\title{
La evaluación como herramienta de trasformación de la práctica docente en tiempo de pandemia, periodo 2020-2021 caso: colegio privado subvencionando italiano Santo Tomas-FROSEP
}

\author{
Elisa Salinas Pérez \\ elisasalinasp@hotmail.com \\ Maestría en Ciencias de la Educación, \\ con énfasis en Investigación Científica. \\ Facultad de Humanidades y Ciencias de la Educación \\ Universidad Nacional de Pilar
}

\section{RESUMEN}

La investigación tiene como objetico determinar las funciones de la evaluación como herramienta de trasformación de la práctica docente en tiempo de pandemia, de los alumnos de la Educación Escolar Básica del Colegio Privado Subvencionando Italiano Santo Tomás-FROSEP. El estudio es carácter descriptivo. Se ha llegado a la conclusión de que la evaluación en el ámbito académico, comparte las funciones de tomar decisiones, todo ello constituye el proceso de evaluación, un proceso complejo, global que cobra su verdadero sentido no en una u otra de sus fases sino en la amplitud de la misma. Los encargados de la evaluación mencionaron la necesidad de la evaluación formativa, orientada a mejorar el aprendizaje de los alumnos, se da también para otros fines tales como la de conocer los conocimientos previos de los alumnos acerca de sus aprendizajes anteriores, para medir y calificar las capacidades individuales para brindar atenciones personalizadas pertinentes a cada caso en particular. Así la evaluación en su dimensión pedagógica, formativa, representa un elemento muy importante del proceso de enseñanza aprendizaje utilizada específicamente cuando el aprendizaje es el objetivo. Destacándose que la principal preocupación de la evaluación en su dimensión formativa es cómo hacer de ella un instrumento de mejora del aprendizaje integrado dentro del proceso de enseñanza-aprendizaje. De esta manera se confirma la hipótesis que plantea que las funciones de la evaluación como herramienta de trasformación de la práctica docente en tiempo de pandemia, está determinada por los diferentes instrumentos y tipo de evaluaciones implementadas por los docentes a los alumnos de la Educación Escolar Básica del Colegio Privado Subvencionado Italiano Santo Tomás-FROSEP.

Palabras clave: evaluación; práctica docente; pandemia; colegio italiano santo tomásFROSEP. 


\title{
Evaluation as a tool for transforming teaching practice in time of pandemic, period 2020-2021 "case: private school subsidizing italian santo tomás-frosep
}

\begin{abstract}
The objective of the research is to determine the functions of the evaluation as a tool for transforming the teaching practice in times of pandemic, of the students of the Basic School Education of the Santo Tomás-FROSEP Italian Subsidizing Private School. The study is descriptive in nature. It has been concluded that evaluation in the academic field shares the functions of making decisions, all this constitutes the evaluation process, a complex, global process that takes on its true meaning not in one or another of its phases but in the breadth of it. Those in charge of the evaluation mentioned the need for formative evaluation, aimed at improving student learning, is also given for other purposes such as knowing the prior knowledge of students about their previous learning, to measure and qualify the individual capacities to provide personalized attention pertinent to each particular case. Thus, evaluation in its pedagogical, formative dimension represents a very important element of the teaching-learning process used specifically when learning is the objective. Emphasizing that the main concern of evaluation in its formative dimension is how to make it an instrument for improving integrated learning within the teaching-learning process. In this way, the hypothesis that proposes that the functions of evaluation as a tool for transforming teaching practice in times of pandemic is confirmed, is determined by the different instruments and types of evaluations implemented by teachers to students of Basic School Education. of the Private Subsidized Italian School Santo Tomás-FROSEP
\end{abstract}

Keywords: evaluation; teaching practice; pandemic; italian school santo tomásFROSEP

Artículo recibido: 15 octubre. 2021 Aceptado para publicación: 18 noviembre 2021 Correspondencia: elisasalinasp@ hotmail.com Conflictos de Interés: Ninguna que declarar 


\section{INTRODUCCIÓN}

Con el Sistema Nacional de Evaluación de la Calidad Educativa, se ha ido incluyendo paulatinamente muestras de los cursos finales de instituciones educativas en los Operativos Nacionales de Evaluación. Dichos operativos, según sus objetivos, permitirían a todos los integrantes de las comunidades educativas contar con la información que facilite la corrección de las dificultades detectadas y conocer la calidad educativa de cada institución y los logros alcanzados por los padres en el proceso educativo de sus hijos.

Según Perrenoud (1990) señala que el éxito y el fracaso escolar no son realidades o apreciaciones objetivas de competencias del estudiante, sino el resultado de cómo se entiende y se valora el proceso y los resultados del aprendizaje de los alumnos. En este sentido se puede afirmar según este autor que los buenos o malos resultados de la evaluación son categorías elaboradas dentro de las instituciones educativas.

La investigación estudia la evaluación como herramienta de trasformación de la práctica docente en tiempo de pandemia, de los alumnos del 3er Ciclo del Colegio Privado Subvencionando Italiano Santo Tomás-FROSEP.

La educación sistemática del país ha sido afectada por una Reforma propuesta e impulsada a través de la política de Gobierno como una necesidad de adecuación y respuestas a las trasformaciones políticas, sociales, económicas y culturales, manifestadas en la sociedad.

En la actualidad, la reforma educativa implica la reorientación de toda actividad educativa, en todos los aspectos y elementos para el logro de la formación integral de los educandos, para así responder a las expectativas de la política nacional, de esta manera se considera a la evaluación de importante relevancia para comprender y mejorar la tarea educativa.

En el ámbito educativo la operación de evaluar consiste en estimar su valor no material; evaluar hace referencia a cualquier proceso por medio del que alguna o varias características de un grupo de alumnos, profesores, materiales, objetivos educativos, reciben la atención de quinen evalúa, analizando y valorando sus características y condiciones en funciones de criterios o puntos de referencia para emitir un juicio relevante a favor de la educación de manera a conocer la marcha de la educación y realizar ajustes necesarios en los procesos educativos. 
La tarea en las aulas de los primeros grados constituye la base de la formación y del desarrollo de los educandos. Es una labor sumamente delicada que requiere, por una parte, buena preparación con sólidos conocimientos teóricos y prácticos y; por otra, la suficiente voluntad para encarar el desafío de la educación con responsabilidad, conciencia y entusiasmo.

Según Arias, Labrador y Gámez (2019). Actualmente muchas realidades evolucionan a tal escala originando que el conocimiento adquiera múltiples connotaciones, lo que deriva en sugestivas perspectivas sobre ellas. La evaluación como tal, desde hace décadas viene siendo entendida por expertos y estudiosos como un proceso álgido posturalmente, puesto que, han surgido desde diversos paradigmas concepciones que a lo largo de su desarrollo histórico han promulgado según su posicionamiento el ser de esta actividad.

\section{REVISION BIBLOGRÁFICA}

\section{La Evaluación educativa}

De acuerdo a Arias, Labrador y Gámez (2019). La evaluación educativa constituye un medio moderador de la enseñanza, mediante ella se puede catalizar acuerdos didácticos que faciliten la mediación y adquisición de conocimientos, así como, realizar ajustes a la diversidad de necesidades, capacidades e intereses del estudiantado a través de reorientaciones que permitan instaurar preceptos válidos y aplicables a futuras evaluaciones contextualizadas y enriquecedoras, para que sean convergentes con el real cumplimiento de su primordial función formadora como es consolidar aprendizajes (Arias, Labrador y Gámez, 2019).

De acuerdo a (Avolio de Cols, 1999). La evaluación es una actividad que realizamos todos los días, sea cual sea el nivel de enseñanza y la modalidad en la que se desarrolle nuestra tarea. Las ideas sobre que es evaluar no son aisladas, se sustentan en otras concepciones básicas, por ejemplo, que es enseñar, cuál es el papel de la escuela, cuál es el rol del docente, cómo aprenden los alumnos, etc.

Los diccionarios, de tipo general consideran que evaluar es tasar, justipreciar, estimar, apreciar el valor de las cosas no materiales, valorar, atribuir cierto valor a una cosa. El elemento clave, en efecto, de la evaluación es el de la valoración de la realidad evaluada. Cuando esa realidad se refiere a las producciones de los alumnos se suele hablar, de evaluación de logros. Cualquier acto de valoración supone la necesidad de un 
patrón o norma que atribuya una valor a esa realidad; a su vez se precisa de algún tipo de información que nos permita la valoración o emisión de un juicio sobre esa realidad para determinados fines; así podemos notar que en la evaluación existen tres elementos que, a la vez, se constituyen en sus etapas: una recogida de información, una valoración de la misma y una toma de decisiones.

La evaluación no es simplemente comprobar resultados. Evaluar es valorar una realidad, que forma parte de un proceso y es el punto de arranque de nuevos aprendizajes y clave para remover los obstáculos que puedan impedir el éxito (García, 1989).

Consideramos importante antes de hablar sobre el concepto de Evaluación, citar algunas definiciones actuales de Evaluación:

"Evaluación es la recogida sistemática de información sobre la naturaleza y la calidad de los objetos conocidos” (Nevo, 1997).

"Evaluación es un proceso que permite profundizar en la naturaleza de lo que estamos evaluando que servirá, para tomar mejores decisiones" (Solabarrieta, 1997).

"La Evaluación de carácter experimental, cuantitativo y sumativo es útil y absolutamente necesaria, sin duda alguna. Pero es un modelo evaluador que tiene su aplicación y máxima utilidad cuando hay que obtener resultados globales, generalizables al conjunto de un sistema y que, por lo tanto, sirven para adoptar medidas, a medio y largo plazo dado el tiempo imprescindible que debe transcurrir desde la toma de datos (al finalizar un proyecto) hasta la obtención de resultados y la posible toma de decisiones" (Nevo, 1997).

Antes de considerar a la evaluación con estas definiciones, se la consideraba como un proceso equiparado al examen y la calificación. Lo fundamental era comprobar la consecución de los objetivos y su función principal era la sumativa, proceso que no permite la revisión de los mismos procesos. Hasta que surgieron diferentes modelos que tienden a romper esta concepción para dar mayor importancia a los procesos en sí. Estos modelos fueron precisados en las definiciones citadas.

Es así que se muestra la necesidad de que en la educación se conozca su situación y se verifiquen los procesos y los resultados, como punto de partida para establecer 
propuestas de mejora en los proyectos, programas o instituciones.

Además, en cada situación de enseñanza-aprendizaje se debe elaborar su propia definición de la evaluación y será también desde su marco teórico o enfoque evaluativo desde el que partirán el objeto y la metodología de su proceso evaluador (Centro de Investigación, Documentación y Evaluación).

A lo largo de las últimas décadas se han propuesto diversos prototipos sobre evaluación, sustentados esencialmente en posicionamientos de lo que se cree cónsono con escenarios educativos del momento o época en particular, desde múltiples perspectivas expertos y estudiosos de esta área del saber han discernido y auspiciando novedosas o renovadas formas de evaluar con la pretensión de superar la errada percepción de considerarla como proceso de instantes únicos registrables y perdurables administrativamente con el fin de certificar y promover.

De acuerdo a Ketele (1988, p. 5) escribía:

La evaluación se reconoce actualmente como uno de los puntos privilegiados para estudiar el proceso de enseñanzaaprendizaje. Abordar el problema de la evaluación supone necesariamente tocar todos los problemas fundamentales de la pedagogía. Cuanto más se penetra en el dominio de la evaluación tanta más conciencia se adquiere del carácter enciclopédico de nuestra ignorancia y más ponemos en cuestión nuestras incertidumbres. Cada interrogante planteada lleva a otras. Cada árbol se enlaza con otro y el bosque aparece como inmenso.

Sin embargo, sigue existiendo, a estas alturas, un alto grado de confusionismo terminológico e ideológico; en demasiadas ocasiones se utilizan, indiscriminadamente, sin la suficiente precisión, términos tales como valoración, medición, evaluación, calificación, etc. e incluso cuando hablamos de evaluación (Arribas, 2017, p.24).

Por estas circunstancias, existe en la literatura especializada abundantes tratados sobre concepciones y modelos de evaluación cuyas exposiciones pretenden superar formas tradicionales de la misma.

En toda evaluación se dan fenómenos básicos, por un lado, la selección restrictiva del objeto a evaluar y por otra parte la emisión de un juicio en la que intervienen 
dimensiones del sujeto u objeto evaluado que no entran en la valoración pero que la condicionan. Esta contaminación se conoce como el "efecto halo", que consiste en la interferencia que produce la opinión que se tiene sobre algunos aspectos de una persona al apreciar cualidades específicas de la misma. En otras palabras, la impresión sobre el todo condiciona la valoración de las partes. Este efecto es inherente a la percepción humana, de ahí que la evaluación objetiva sea a todas luces imposible. Un claro ejemplo de esto sería que se evalúe a un alumno en función de su correcta presentación o buenos modales. Hablar de valoración en evaluación implica distinguir criterios de referencia, no sólo en quien realiza el juicio sino también como éste se lleva a cabo (Arias, Labrador y Gámez, 2019, p.3).

\section{La Evaluación y el propósito del aprendizaje}

Si bien la evaluación tiene un lugar de importancia en la agenda didáctica, ha sido en cierta forma adquirida como resultado de una "patología", ya que el profesor transformó muchas de sus prácticas en función de la evaluación, tornándose ésta en un estímulo más importante que el propio aprendizaje (Careaga, 2001).

Litwin (1998) sostiene que, en las prácticas de enseñanza, la actitud evaluadora invierte el interés de conocer por el interés por aprobar en la medida que se estudia para aprobar y no para aprender. Es precisamente el propio profesor que cuando enseña un tema considera relevante resalta su importancia manifestando que será evaluado y de esa forma va estructurando toda la situación de enseñanza por la propia situación de evaluación. Sin embargo, esta práctica "patológica" puede verse modificada si los docentes recuperan el lugar de la evaluación como el espacio donde se genera la información acerca de la calidad de su propuesta de enseñanza. Otra característica que presenta la evaluación es que se la relaciona casi exclusivamente con procesos de medición, de acreditación o de certificación y rara vez con el proceso de toma de conciencia de los aprendizajes adquiridos o con las dificultades de la adquisición, de la comprensión o de la transferencia de algunos temas o problemáticas (Careaga, 2001).

\section{Visión retrospectiva de la evaluación}

Debido a que la acepción del término históricamente ha sido influenciada por las múltiples perspectivas que se han presentado en la sociedad del conocimiento, resulta determinante para este trabajo, realizar en principio un recorrido en la línea del tiempo sobre este particular, para destacar algunos aportes de personalidades y ciertos 
acontecimientos, para luego analizar una serie de conceptualizaciones, abordar algunos de los modelos de evaluación y así estudiar las tendencias de evaluación.

Sobre dichas tendencias se muestran y explican un conjunto de diagramas contentivos de información sobre hechos históricos y autores de una serie de aportaciones en diferentes épocas y momentos de la evolución representada por actividades y procesos concernientes con lo que se conoce como evaluación, esto en función de un estudio secuencial elaborado y expuesto por Mateo y Martínez, 2008, mencionado por Arias, Labrador y Gámez, 2019, p.4).

Hablar y desentrañar sobre la historia de la evaluación derivaría en la elaboración de un libro, puesto que habría que tomar en cuenta sucesos acaecidos desde tiempos remotos (AC y DD), pasando por la clasificación de las era que se realiza desde la historia universal; por lo que para el presente trabajo se considera a partir del siglo anterior.

A continuación se muestra la Fig. $\mathrm{N}^{\circ} 1$ que versa sobre el origen o génesis de la evaluación.

\subsection{Era del Génesis}

Figura $\mathbf{N}^{\circ}$ 1: Retrospectiva de la Evaluación. Época del Génesis.

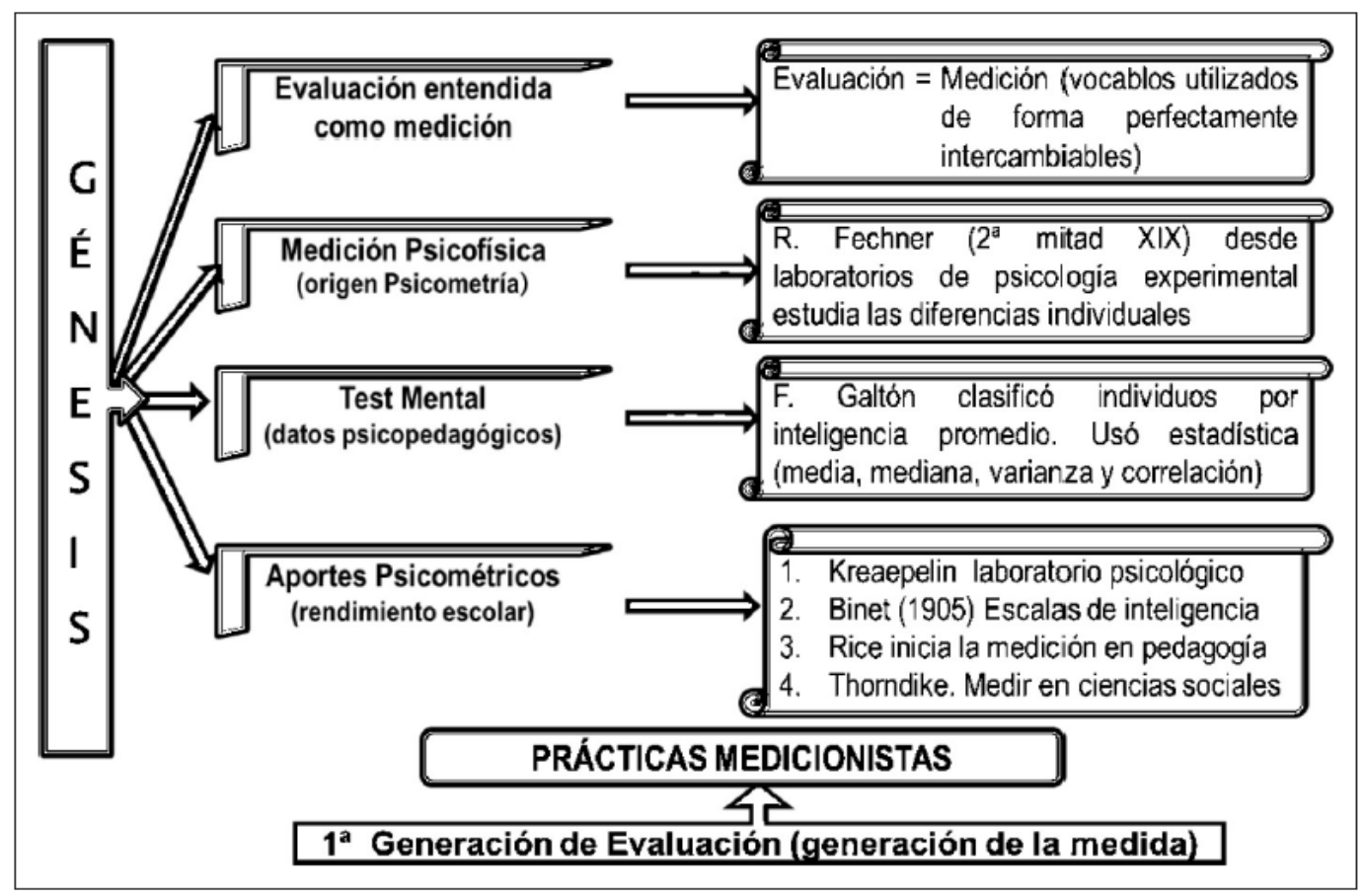

Fuente: (Arias, Labrador y Gámez, 2019). 
Este esquema ubica la evaluación desde períodos remotamente precedentes como cuando en la China Imperial se utilizaron procedimientos para la escogencia de funcionarios, o en el siglo XV de la edad media cuando en las universidades se introducen los exámenes orales públicos ante el maestro y un tribunal como medios para certificar conocimientos.

Sin embargo, para el interés del presente trabajo se decide iniciar desde el siglo XIX resaltando el trabajo de Fechner, quien desde los laboratorios de psicología experimental estudia las diferencias individuales de los sujetos, a lo que llamó medición psicofísica. Según los estudiosos de esta temática a este hecho se considera como el origen de la psicometría.

En este aspecto (Galtón, mencionado por Arias, Labrador y Gámez, 2019) clasificó los individuos según su inteligencia promedio mediante el uso de algunas medidas estadísticas para poder establecer comparaciones entre ellos; es cuando se desarrollan los test mentales, y ayudo a Pearson en el estudio de las asociaciones de las diferencias individuales.

Estos estudios se extendieron rápidamente a países de Europa y Estados Unidos de América, derivando en aportes psicométricos sobre el rendimiento estudiantil. Kreaepelin en su laboratorio psicológico realizó mediciones de la memoria. Binet en 1905 logró construir las primeras escalas para medir la inteligencia.

Rice, a quien se considera el precursor de la medición en pedagogía, llegó a dar los primeros aportes para que Thorndike la colocará en vigencia a través de la medición en las ciencias sociales.

Esta época denominada la del génesis se caracteriza por la relevancia que e importancia de la medición con lo que actualmente se le llama evaluación. Equivalente a lo que se considera como la primera generación de la evaluación o generación de la medida.

Así mismo es considerado como el periodo "pretyleriano" por la mayoría de expertos, vigente hasta mediados del siglo pasado, fundamentado en la medición con el uso básico de test y pruebas para determinar el dominio de conocimientos, donde el evaluador era considerado un experto en la aplicación de medios de evaluación.

Esto permitía la obtención de datos numéricos para poder establecer comparaciones de puntajes de cada sujeto con respecto al grupo que pertenece, así como cotejos entre sujetos, por lo que se considera normativa (Guba y Lincoln, 1989, mencionado por 
Arias, Labrador y Gámez, 2019, p.5).

Otro momento que debe tenerse presente en la historia de la evaluación es la época del dinamismo, donde se orienta la evaluación hacia procesos más dinámicos que incluyen otros elementos como el currículum, considerándolo un gran plan organizado en función de objetivos establecidos. Maniobra que aparte de objetivos, contenidos y procedimientos instruccionales contenía estrategias de evaluación.

Por lo que actualmente la comunidad en general considera que este es el momento en que nace la evaluación. La cual fue definida en principio "como algo que determina si han sido alcanzados ciertos objetivos" (Stufflebeam y Shinkfield, 1989. p.34, Gmencionado por Arias, Labrador y Gámez, 2019, p.5).

Esto desencadeno en que diversos expertos colaboraran con quienes elaboraban currículos en los análisis de relación con el objeto evaluado. Así, de lo resultante del estudio de los objetivos se estructuraba el currículo. Término que asume un rol protagónico hasta los actuales momentos, como el eje principal sobre el cual giran los procesos educativos. En esta época se puede ubicar el modelo de evaluación como medición.

\subsection{Era del Dinamismo}

En la siguiente $\underline{\text { Fig. }} \mathrm{N}^{\circ} 2$ se esboza a grandes rasgos lo concerniente a la época del dinamismo.

Figura $\mathbf{N}^{\circ}$ 2: Retrospectiva de la Evaluación. Época del Dinamismo.

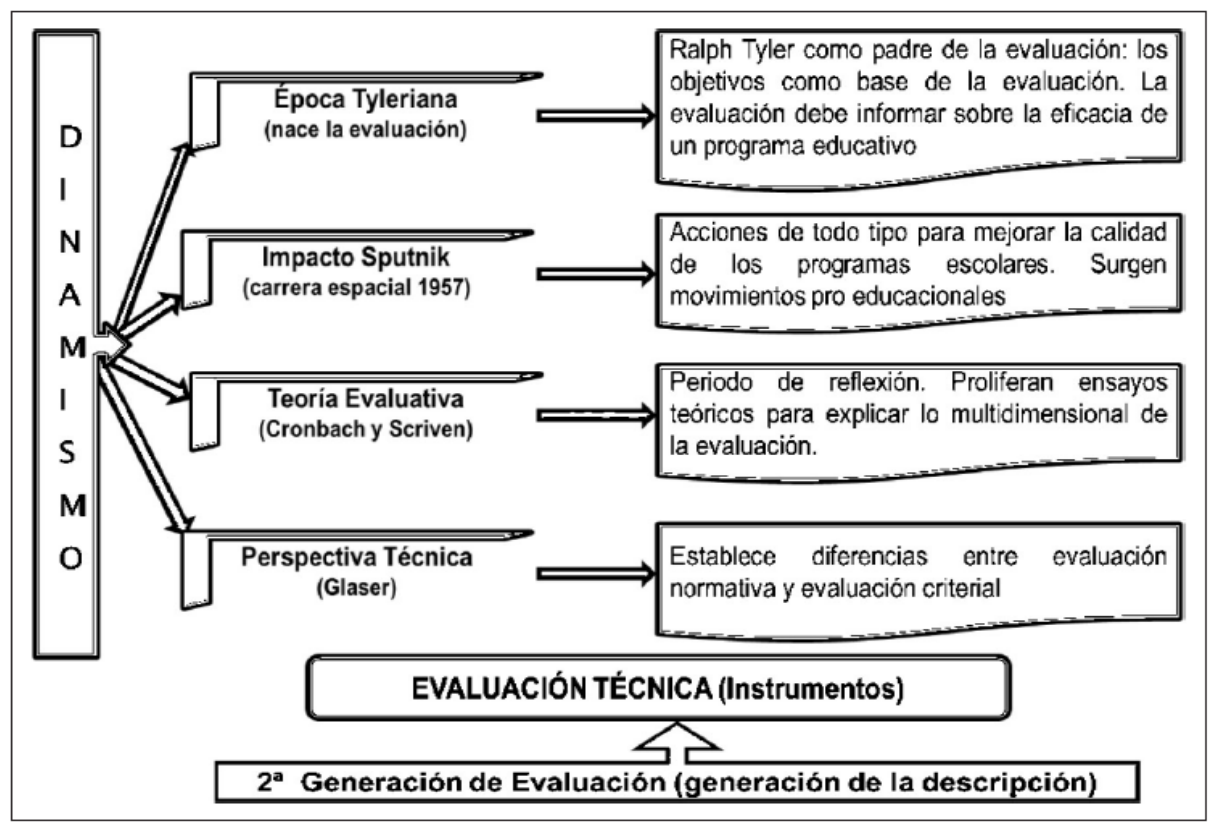

Fuente: (Arias, Labrador y Gámez, 2019, p. 6). 
Esta representación en principio centra la atención en el trabajo de Ralph Tyler (considerado el padre de la evaluación), el cual destaca que los objetivos previamente instituidos que se fijan constituyen la base de la evaluación.

Básicamente Tyler instaura que los objetivos del programa o proceso deben formularse en términos de conducta observable, y que el papel de la evaluación era determinar el cambio de conducta ocurrido, para así establecer lo eficaz de dicho proceso (Arias, Labrador y Gámez, 2019, p 6)

Para este momento en los Estados Unidos de América se inicia una serie de críticas sobre la eficacia de su sistema educativo, auspiciado para muchos por un evento determinante como lo fue la carrera hacia la conquista del universo, dado que la Unión Soviética logra en el año 1957 ser el primer país en lanzar un satélite al espacio (el Sputnik). Esto llevó a generar y desarrollar proyectos escolares en pro de mejorar la educación, igualmente se originan las intervenciones administrativas a las instituciones. Paralelamente se inicia un periodo cargado de reflexiones con aportes teóricosconceptuales redundantes en las bases metodológicas de la evaluación.

Aquí sobresalen los trabajos de Cronbach y Scriven, el primero destaca de la evaluación su concepto, funciones y metodología al incorporar las nociones de evaluaciones previas al final de un proceso, la toma de decisiones en función de la evaluación y centrar el interés en valorar las estructuras de los programas.

Por su parte, Scriven introduce la función formativa y sumativa de la evaluación, dando lugar a toda una teoría evaluativa.

Glaser participa con una visión más técnica estableciendo diferencias primordiales entre la medición de objetivos tomando como referencia la norma, en la cual se determinan posiciones relativas del puntaje de un sujeto en cuanto a lo que es normativo en su grupo, y la medición que toma como referencia un criterio, lo cual permite comparaciones absolutas.

Este periodo llamado del dinamismo, la evaluación adquiere carácter técnico ante el florecimiento de aportes teóricos que impulsaron el diseño de procedimientos e instrumentos, por ello, también se considera como la segunda generación de la evaluación llamada generación de la descripción (Arias, Labrador y Gámez, 2019, p 6) 
La segunda generación en contraposición con la anterior presenta una perspectiva renovada al considerar no solo al sujeto evaluado, sino también otros compendios como el currículo, programas, estrategias, materiales instruccionales entre otros.

También se establece el modelo de Planificación curricular tecnológico, con lo cual se hace énfasis en la selección, esquematización y organización de contenidos, sobre todo en las estrategias para obtener información y valorar el logro de los objetivos.

Se le otorga el nombre de la generación de la descripción porque el evaluador o quien evalúa, se constituye en un descriptor y medidor que colabora con la selección y formulación de objetivos educativos, proporcionando referentes válidos para considerar y establecer fortalezas y debilidades curriculares (Arias, Labrador y Gámez, 2019, p 6).

\subsection{Era de la Profesionalización}

Seguidamente se presenta la época de la profesionalización en la cual surgen variados modelos con la pretensión de reglamentar procederes, acciones y actuaciones evaluativas.

Cabe resaltar que este auge de propuestas teóricas sobre modelos de evaluación hace que se constituya en el periodo que mayoritariamente enriquece la literatura existente en esta temática. El aspecto resaltante de esta era es que se incluye el juicio como componente de la evaluación.

A continuación se muestra la Fig. $N^{\circ} 3$ correspondiente a la época de la profesionalización.

En el diagrama se observan una serie de autores con sus respectivos aportes, unos derivados de la postura de Cronbach y otros de Scriven.

Inicialmente se tiene a Parlett y Hamilton quienes mediante el modelo que llamaron "evaluación iluminativa" afirman que en el proceso evaluativo se debe descubrir y documentar a todos los actores que participen en el mismo, para así comprender y argumentar lo relevante de lo que se pretende abarcar; indican que existen cuatro elementos a considerar: lo que se va a evaluar (objeto), la metodología seguida (el cómo), la estructura que subyace y los valores implícitos. 
Figura $\mathbf{N}^{\circ}$ 3: Retrospectiva de la Evaluación. Época de la Profesionalización.

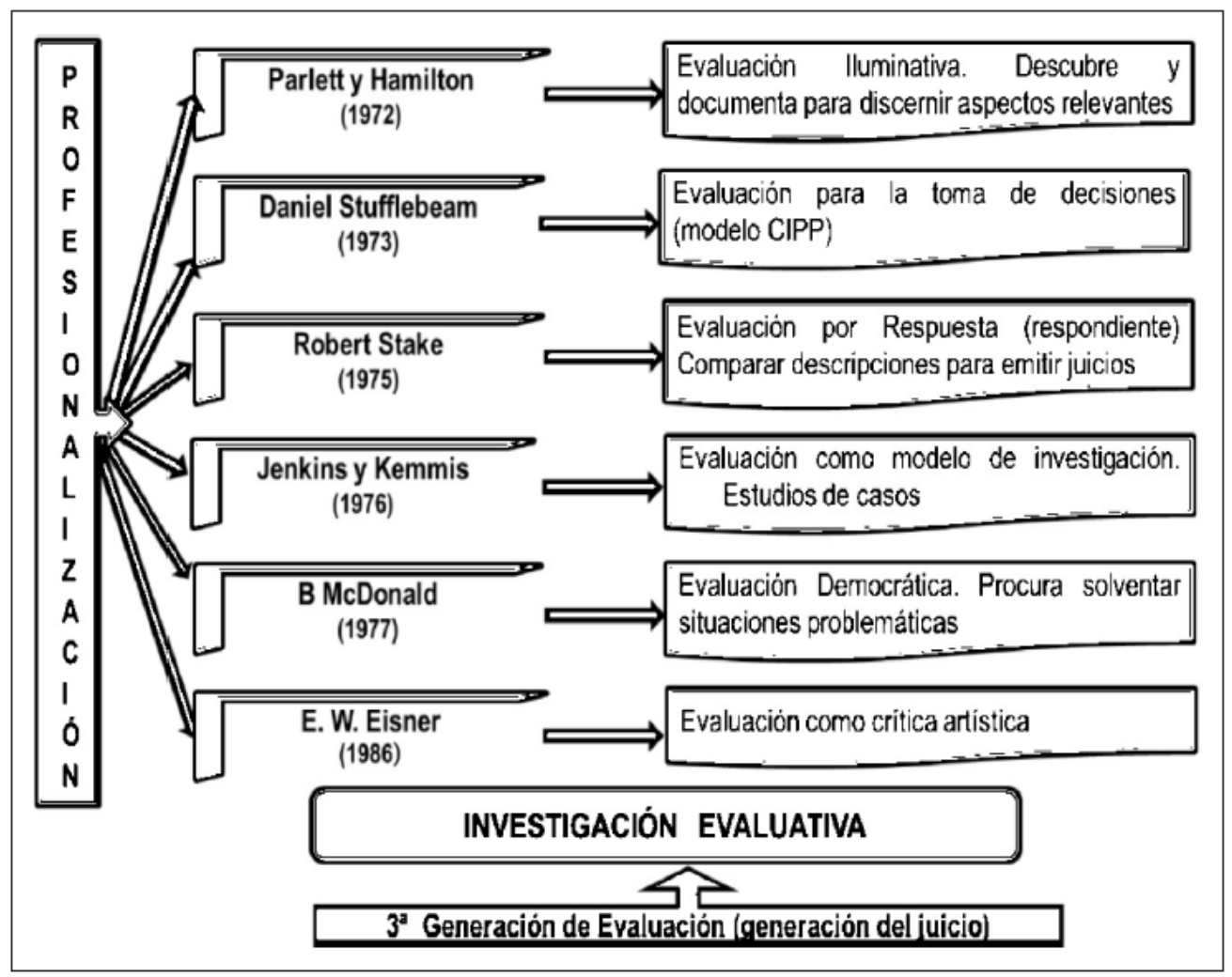

Fuente: (Arias, Labrador y Gámez, 2019, p 6).

Daniel Stufflebeam es considerado el principal proponente de esta era y padre moderno de la evaluación, al considerarla como un proceso sistémico mediante el cual se obtiene información útil para la toma de decisiones. Introduce el modelo CIPP, siglas de Contexto, Insumos, Proceso y Producto, cuatro elementos que deben considerar en la evaluación para poder tomar decisiones apoyadas en la información que proporcionan.

Por su parte, Robert Stake presenta su modelo de "evaluación respondiente", dado que da respuesta a cuestionamientos reales de los involucrados en el proceso evaluativo de un programa educativo desarrollado. Lo primordial del modelo es la comparación de descripciones con estándares para posteriormente emitir juicios valorativos.

Jenkins y Kemmis definen e introducen las prácticas investigativas como evaluación, en sí definen el modelo de investigación apoyados en el estudio de casos, al tomar este como una práctica evaluativa propia.

Otro de los modelos es el de "evaluación democrática" propuesto por McDonald a través del cual se pretende solventar situaciones problemáticas, esta visión le otorga carácter político a la evaluación. 
Eisner con orientación cualitativa propugna el modelo de evaluación conocido como "crítica artística", el cual entiende la educación como una creación artística, donde quien evalúa se percibe como un experto que reflexiona lo que observa para interpretar y valorar lo que aprecia.

Este periodo de la profesionalización fue profundamente enriquecido por la aparición de modelos evaluativos, pero el gran avance consistió en que logró colocar a la evaluación en el espacio de la investigación, dando origen a la investigación evaluativa.

A todos estos acontecimientos es lo que se conoce como la tercera generación de evaluación, llamada la generación del juicio. Dicha generación introduce la emisión de juicios como parte o componente de la evaluación con el propósito de tomar decisiones. En esta época de la profesionalización se descubrió la no existencia en periodos anteriores de medios que facilitara a los evaluadores el intercambio de saberes para promover entre ellos una formación adecuada en el tema evaluativo. Por ello es en esta era que surge la evaluación educativa como una profesión distinta a la investigación educativa, lo que da lugar a disertaciones a sus alcances (Lukas y Santiago, 2004, mencionado por Arias, Labrador y Gámez, 2019, p 6).

\subsection{Era de la Integración}

Finalmente se considera la época de la integración que tiene lugar a partir de 1990 considera la pluralidad de enfoques en busca de una postura que resolviera esta situación, muchos fueron de la idea que sería el modelo respondiente y el enfoque constructivista.

Sin embargo, el trascurso del tiempo deja constancia en primer término de los esfuerzos de los actores teóricos por tratar de crear conciencia entre los evaluadores de que el acto evaluativo debe redimensionarse en procura de que la evaluación cumpla realmente con sus funciones de orientadora y colaborativa para alcanzar la optimización de los objetos sujetos a sus prácticas de valoración. Y en segundo, lograr que los evaluadores interioricen estas nuevas posturas. 
La Fig. $\mathrm{N}^{\circ} 4$ muestra al igual que los anteriores en forma esquematizada este periodo.

Figura $\mathbf{N}^{\circ}$ 4: Retrospectiva de la Evaluación. Época de la Integración.

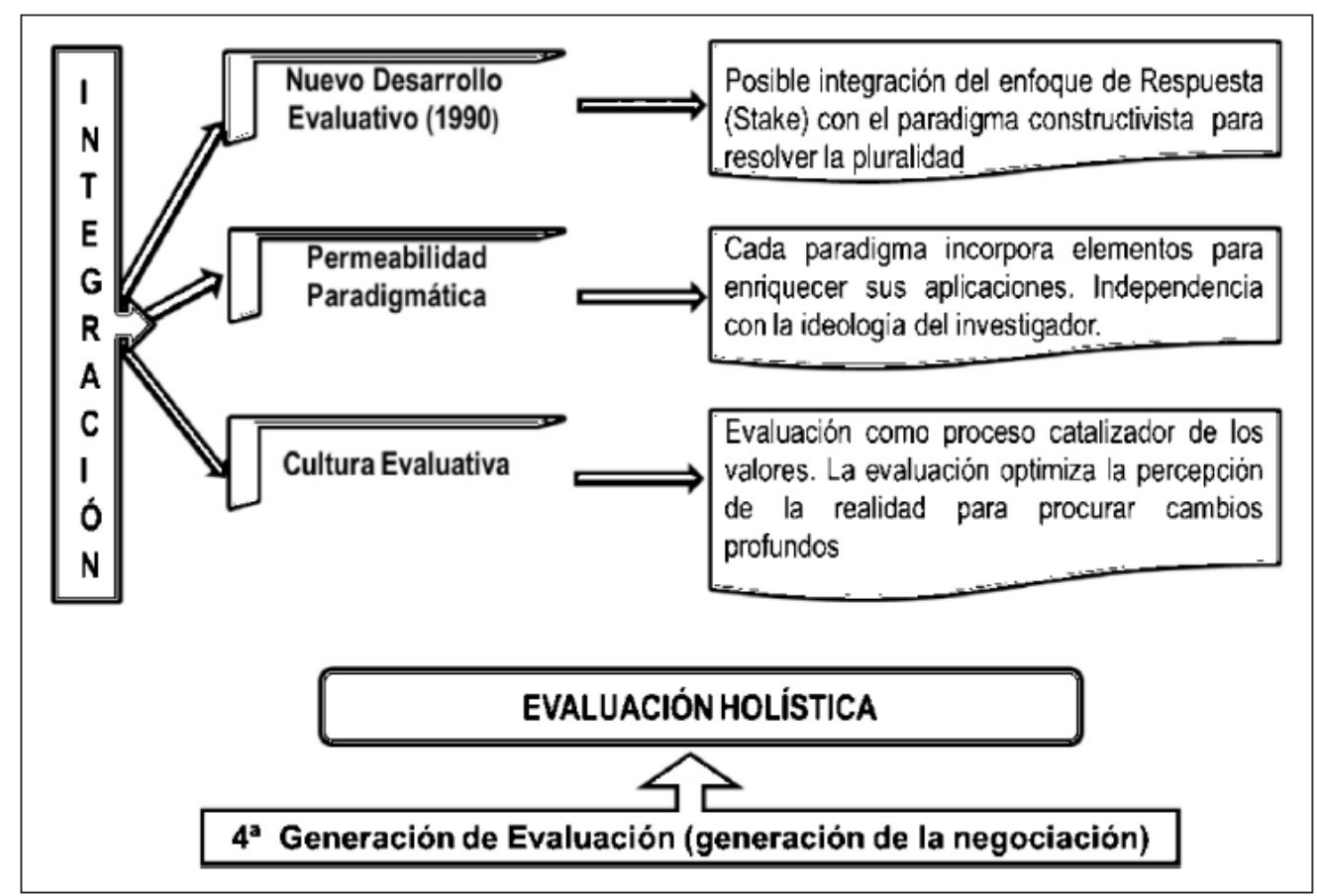

Fuente: (Arias, Labrador y Gámez, 2019, p 7).

De acuerdo con la ilustración anterior, en primera instancia lo que distingue a esta época es el esfuerzo de un nuevo desarrollo evaluativo mediante la posible integración del modelo de repuesta con el gran modelo constructivista, donde se va más allá de las descripciones para ir a las construcciones significativas de los sujetos evaluados.

En segundo término, se considera la permeabilidad paradigmática en función del método, donde cada paradigma incorpora elementos propios para enriquecer sus aplicaciones manteniendo la independencia ideológica de quien desarrolla el proceso, lo que sí debe tenerse presente es el nivel de estructuración y la tipología organizativa que caracteriza la realidad estudiada, donde el evaluador deja de lado su rol de juez para convertirse en un investigador de los procesos (Arias, Labrador y Gámez, 2019, p 7).

Todo lo anterior desemboca en la cultura evaluativa de entender los procesos de evaluación más allá de lo metodológico o técnico, para ejercer funciones de catalizador axiológico, que sensibilice la percepción de la realidad procurando cambios profundos.

Dicha cultura debe constituir un proceso generador y constructor de valores que deben integrarse en favor de lo evaluado. En este caso, la evaluación debe entenderse desde la construcción social y personal del evaluador. 
Los hechos anteriores son los que caracterizan la cuarta generación de evaluación llamada la generación de la negociación. Para otros considerada la generación constructivista y respondiente producto de acciones interactivas de negociación que involucra a todos los actores comprometidos en el acto evaluativo.

Por lo expuesto hasta ahora, se puede concluir que en principio la evaluación fue entendida como procesos de medición para ir evolucionando a concepciones más sociales y humanizantes, contentivas de aportes teóricos-metodológicos de una serie de modelos de evaluación que han surgido, y que seguidamente se diserta sobre algunos de ellos (Arias, Labrador y Gámez, 2019, p 7).

\section{METODOLOGIA}

La investigación se enmarca en un estudio de tipo descriptivo correlacional, con la finalidad de describir características propias de las variables en estudio como son las funciones evaluativas más aplicada en la práctica docente, los diferentes instrumentos evaluativos considerados y los tipos de evaluaciones aplicado en tiempo de pandemia.

Con diseño no experimental, en cuanto a la cronología en el tiempo, esta investigación es de corte trasversal o transaccional, ya que se estudió en un periodo de 2020-2021.

Y abordó una metodología de enfoque mixto.

\section{ANÁLISIS Y DISCUSIÓN}

Luego del análisis de los instrumentos de recolección de datos aplicado a los diferentes estamentos de la institución en estudio, a continuación, se presenta los resultados de la investigación conforme a las variables estudiadas:

Los resultados de las puntuaciones resultan de la siguiente sumatoria: Puntuación General: 14 preguntas por 124 sujetos encuestados y 3 niveles de opción respuesta. Totalizando así: 5.208 puntos posibles, si es que todos optan por el nivel máximo 3, en su respuesta.

Las puntuaciones se agrupan por dimensión, subdimensión e indicador, según la opción, dónde: 1: Nunca 2: A veces; 3: Siempre.

\section{- Respecto a la variable: Funciones evaluativas}

Instrumento: Cuestionario dicotómico

Para el efecto, se ha determinado establecer los siguientes subdimensiones: Funciones evaluativas y en torno a ellos enunciaron 6 preguntas con sus 3 opciones de respuesta. 
Figura 1. Distribución de los puntajes en porcentajes, relacionados a la Dimensión1:

"Funciones evaluativas", agrupadas por subdimensiones según opciones de respuestas.

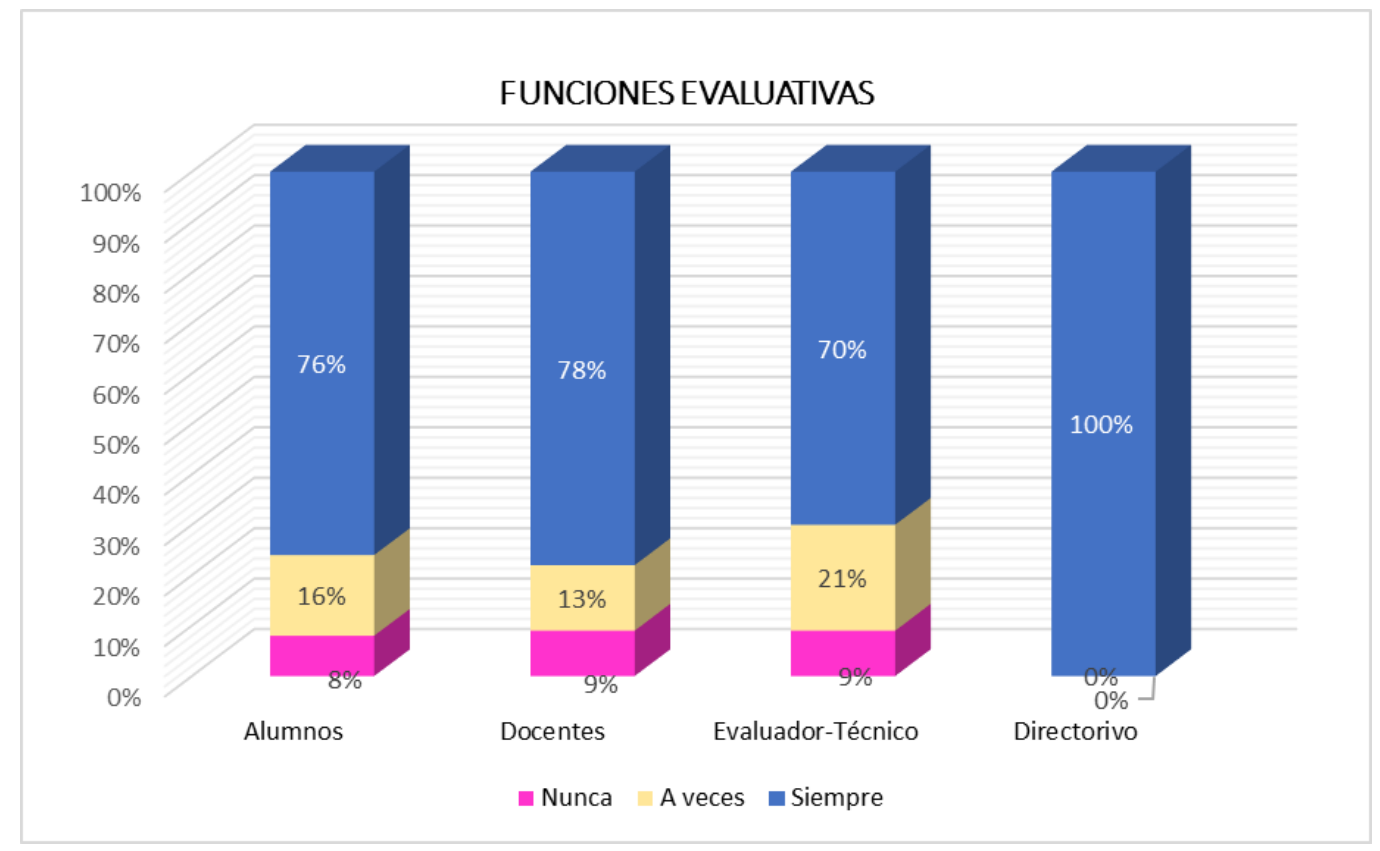

Al efectuar un análisis enfocado a la subdimensión: funciones evaluativas y sus indicadores: (Funciones diagnóstica, sumativa, formativa; Actuación personal de atención a los alumnos; Coordinación de actividades con otros maestros y Comunicación con los padres o tutores); según la escala de valoración, se observa un rango de afirmativo de tres niveles en primer lugar la opción siempre el porcentaje más relevante que corresponde a la estructura de cada nivel y los demás indicadores en porcentajes positivos interiores, evidenciándose un nivel alto de la escasa respecto a la variable influencia de la las funciones evaluativas.

La evaluación diagnóstica permite conocer el punto de partida con el fin de orientar el subsiguiente proceso en una determinada dirección o certificador, y constatar el grado de consecución de los objetivos previamente determinados con el fin de avalar, clasificar, discriminar y si es necesario revisar, rehacer y retomar medidas correctivas necesarias. La evaluación en el ámbito académico, comparte las funciones de tomar decisiones, todo ello constituye el proceso de evaluación, un proceso complejo, global que cobra su verdadero sentido no en una u otra de sus fases sino en la amplitud de la misma.

Por evaluación asumimos la definición que proponen Gairín, Carbonell, Paredes, y Santos, (2009, p. 8): “el proceso de recogida, análisis e interpretación de resultados 
con el fin de valorarlos y que conlleva una toma de decisiones".

Partiendo de esta definición y haciendo abstracción de las innumerables definiciones del concepto de evaluación, tres son los elementos fundamentales que la caracterizan: primero, recogida de información, medición-; segundo, valoración, interpretación de esa información y tercero, toma de decisiones en función de los dos primeros.

\section{- Respecto a la variable: Instrumentos evaluativos}

Figura 2. Distribución de los puntajes en porcentajes, relacionados a la Dimensión 2: Instrumentos evaluativos, agrupadas por subdimensiones según opciones de respuestas.

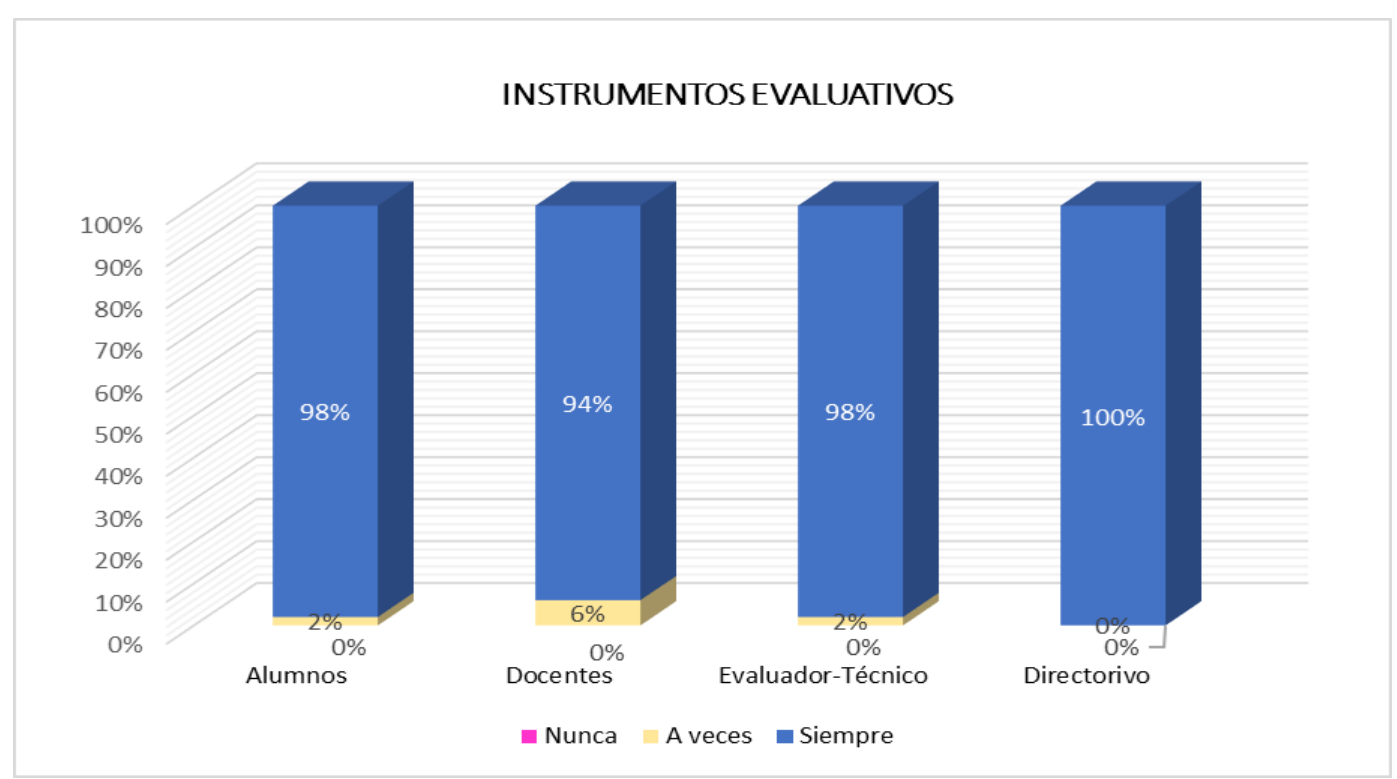

$\mathrm{Al}$ efectuar un análisis enfocado a la variable: dificultades de aprendizaje y sus indicadores: pruebas escritas: de selección múltiples, alternativas contante y pareo; Pruebas orales: lista de cotejo, registro anecdótico, guía de entrevista; pruebas prácticas: portafolio de evidencias, y mapas conceptuales e interacciones constantes, destacándose que la evaluación en su dimensión pedagógica, formativa, representa un elemento muy importante del proceso de enseñanza aprendizaje utilizada específicamente cuando el aprendizaje es el objetivo.

Contrastando con la teoría (Villardón, 2006, pp. 61-62, en Gairín, 2008, p.73), expone la evaluación en su función sumatoria como evaluación de competencias y la evaluación formativa como evaluación para el desarrollo de competencias son dos enfoques complementarios y necesarios de la evaluación de los aprendizajes, que conducen a una concepción global de lo que tiene que ser la evaluación (...) como elemento de formación competencial. 


\section{- Respecto a la variable: Tipos de evaluación}

Figura 3. Distribución de los puntajes en porcentajes, relacionados a la Dimensión 3:

“Tipos de evaluación”, agrupadas por subdimensiones según opciones de respuestas

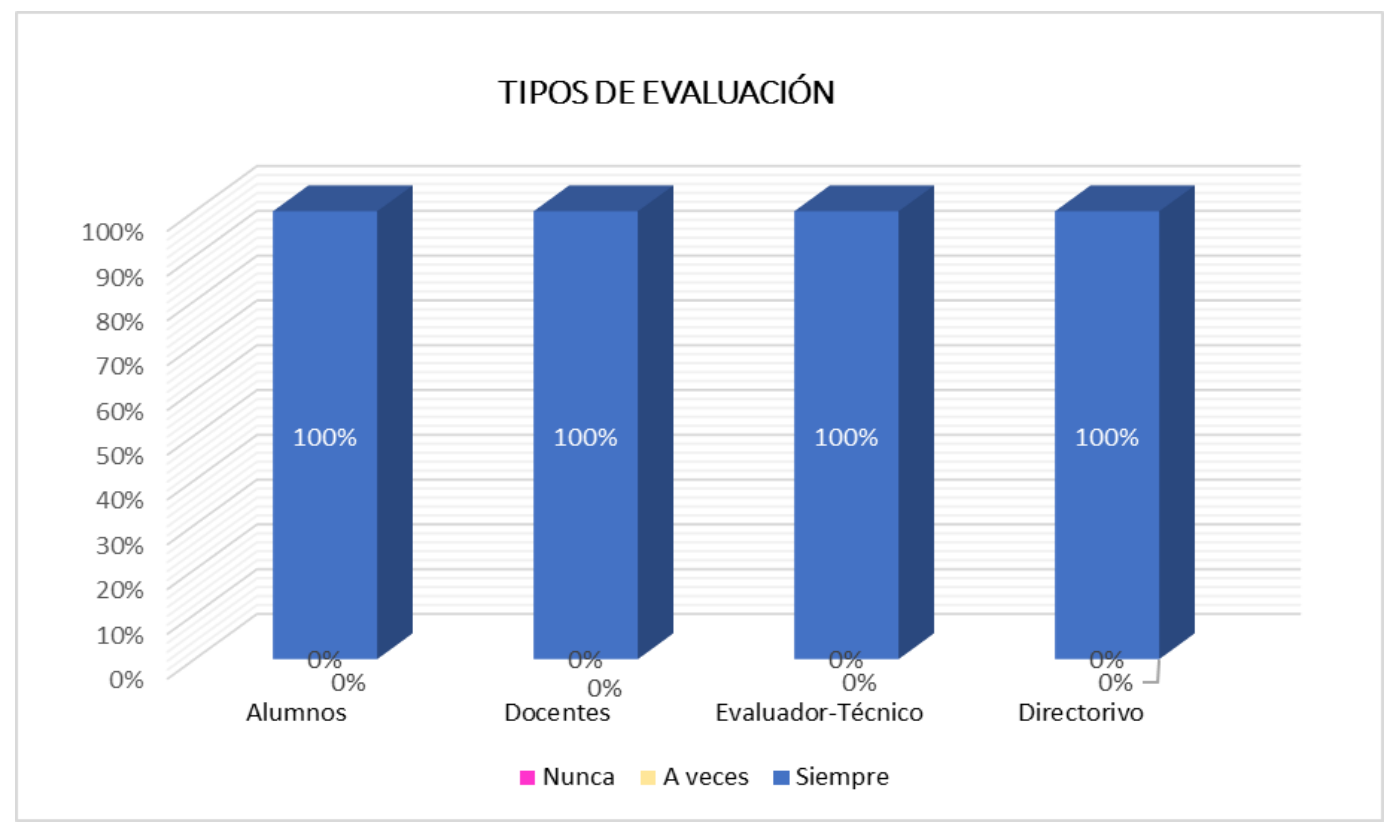

Al efectuar un análisis enfocado a la subdimensión "Tipos de evaluación”, con sus indicadores: Autoevaluación, Coevaluación y Evaluación Unidireccional, según la escala de valoración un nivel adecuado del 100\% de todos los estamentos. De acuerdo a los indicadores mencionados se utilizan todos los tipos de evaluaciones.

De acuerdo a (Avolio de Cols, 1999). La evaluación es una actividad que realizamos todos los días, sea cual sea el nivel de enseñanza y la modalidad en la que se desarrolle nuestra tarea. Las ideas sobre que es evaluar no son aisladas, se sustentan en otras concepciones básicas, por ejemplo, que es enseñar, cuál es el papel de la escuela, cuál es el rol del docente, cómo aprenden los alumnos, etc.

Los diccionarios, de tipo general consideran que evaluar es tasar, justipreciar, estimar, apreciar el valor de las cosas no materiales, valorar, atribuir cierto valor a una cosa. El elemento clave, en efecto, de la evaluación es el de la valoración de la realidad evaluada. Cuando esa realidad se refiere a las producciones de los alumnos se suele hablar, de evaluación de logros. Cualquier acto de valoración supone la necesidad de un patrón o norma que atribuya una valor a esa realidad; a su vez se precisa de algún tipo de información que nos permita la valoración o emisión de un juicio sobre esa realidad para determinados fines; así podemos notar que en la evaluación existen tres elementos 
que, a la vez, se constituyen en sus etapas: una recogida de información, una valoración de la misma y una toma de decisiones.

La evaluación no es simplemente comprobar resultados. Evaluar es valorar una realidad, que forma parte de un proceso y es el punto de arranque de nuevos aprendizajes y clave para remover los obstáculos que puedan impedir el éxito (García, 1989).

\section{CONSIDERACIONES FINALES}

Respecto a las funciones evaluativas más aplicada en la práctica docente en tiempo de pandemia, se resumen que la evaluación diagnóstica de los alumnos permite obtener un panorama de la situación actual del alumno lo que permitirá fortalecer las áreas necesaria, por ello todos los docentes encuestados afirmaron que, efectivamente, consideran de suma importancia dicha evaluación diagnostica de sus alumnos.

La mayoría de los alumnos siempre consideran importante la evaluación diagnóstica,

siendo que permite conocer su situación actual, así también la evaluación formativa, mientras.

Así los encargados de la evaluación mencionaron la necesidad de la evaluación formativa, orientada a mejorar el aprendizaje de los alumnos, se da también para otros fines tales como la de conocer los conocimientos previos de los alumnos acerca de sus aprendizajes anteriores, para medir y calificar las capacidades individuales para brindar atenciones personalizadas pertinentes a cada caso en particular.

Los diferentes instrumentos evaluativos considerados en la práctica docente en tiempo de pandemia, se aplican las pruebas escritas y también los trabajaos prácticos; $\mathrm{y}$ en algunos casos las pruebas orales.

Así la evaluación en su dimensión pedagógica, formativa, representa un elemento muy importante del proceso de enseñanza aprendizaje utilizada específicamente cuando el aprendizaje es el objetivo.

La evaluación diagnóstica permite conocer el punto de partida con el fin de orientar el subsiguiente proceso en una determinada dirección o certificador, y constatar el grado de consecución de los objetivos previamente determinados con el fin de avalar, clasificar, discriminar y si es necesario revisar, rehacer y retomar medidas correctivas necesarias. La evaluación en el ámbito académico, comparte las funciones de tomar decisiones, todo ello constituye el proceso de evaluación, un proceso 
complejo, global que cobra su verdadero sentido no en una u otra de sus fases sino en la amplitud de la misma.

Los diferentes tipos de evaluaciones aplicado por los docentes en tiempo de pandemia. Todos los docentes y directores consideran muy importante la evaluación de los alumnos.

Todos los docentes, siempre al evaluar utiliza la evaluación unidireccional (docente $\rightarrow$ alumnos) como también la evaluación bidireccional (docente $\leftrightarrow$ alumno); y a veces utilizan la autoevaluación (evaluarse a sí mismo), esto sucede al final del año sobre todo en consenso de profesores.

Los docentes siempre practican la autoevaluación (evaluarse a sí mismo).

Destacándose que la principal preocupación de la evaluación en su dimensión formativa es cómo hacer de ella un instrumento de mejora del aprendizaje integrado dentro del proceso de enseñanza-aprendizaje, la preocupación de la evaluación en su dimensión certificadora en el ámbito académico es precisamente su validez, fiabilidad y practicidad a la hora de constatar la consecución de los objetivos y competencias académicas y profesionales orientada a la mejora del aprendizaje del alumno.

De esta manera se confirma la hipótesis que plantea que las funciones de la evaluación como herramienta de trasformación de la práctica docente en tiempo de pandemia, está determinada por los diferentes instrumentos y tipo de evaluaciones implementadas por los docentes a los alumnos de la Educación Escolar Básica del Colegio Privado Subvencionado Italiano Santo Tomás-FROSEP.

\section{REFERENCIAS BIOGRÁFICAS}

Arias Lara, S.A; Labrador L. N y Gámez Valero, B (2019). Modelos y épocas de la evaluación educativa. Universidad de los Andes, Universidad Experimental Nacional del Táchira San Cristóbal, Venezuela. Educere, vol. 23, núm. 75, pp. $307-322,2019$

Arias Lara, S.A; Labrador L. N y Gámez Valero, B (2019). Modelos y épocas de la evaluación educativa. Universidad de los Andes, Universidad Experimental Nacional del Táchira San Cristóbal, Venezuela. Educere, vol. 23, núm. 75, pp. 307-322, 2019

Arias, F. (2006). El Proyecto de Investigación $6^{\mathrm{a}}$ Edición. Editorial: Episteme. Venezuela 
Arribas Estebaranz, J. Mª (2017). La evaluación de los aprendizajes. Problemas y soluciones Profesorado. Revista de Currículum y Formación de Profesorado, vol. 21, núm. 4, septiembre-diciembre, 2017, pp. 381-404 Universidad de Granada Granada, España.

Avolio De Cols, S, (1998) Los proyectos para el trabajo en el aula: interrogantes básicos: ¿por qué?, ¿para qué?, ¿cómo? Y ¿ qué? Tomo 2. Marimar. Bs As.

Avolio De Cols, S.(1999) Dir. Curso de Educación a Distancia. La Evaluación en el marco de los proyectos de aula. Buenos Aires. Marymar para CEDOC.

Bermon, L. (2012). Dirección Nacional de innovación Académica de la Universidad Nacional de Colombia. Modelos. Recuperado el 17 de Marzo del 2015 de http://www.virtual.unal.edu.co/cursos/sedes/manizales/4060010/lecciones/Capitulo1/modelo.htm

Blanco Gutiérrez, Oscar enrique. (1995). Estrategias Metodológicas y procedimentales de Evaluación que utilizan los Profesores del Componente Docente del P.E.B.I. de la ULA-Táchira. Tesis Doctoral no Publicada. Universidad de Los AndesUniversitat Rovira i Virgili.

Careaga, A. (2001). La evaluación como herramienta de transformación de la práctica docente. Educere, vol. 5, núm. 15, octubre-diciembre, 2001, pp. 345-352 Universidad de los Andes Mérida, Venezuela.

Díaz Barriga Arceo, (2000), Frida y Hernández, Gerardo. Estrategias docentes para un aprendizaje significativo. Docente del Siglo XXI: como desarrollar una práctica docente competitiva. Colombia. McGraw - Hill.

Escudero Escorza, T (2003), Desde los test hasta la investigación evaluativa actual. Un siglo, el XX, de intenso desarrollo de la evaluación en educación

Ezequiel, Ander-Egg, (1996) La planificación educativa: conceptos, métodos, estrategias y técnicas para educadores. Buenos Aires. Magisterio del Río de la Plata.

Flórez Ochoa, R, (2000), Evaluación Pedagógica y Cognición. Docente del Siglo XXI: como desarrollar una práctica docente competitiva. Colombia. McGraw - Hill.

García Hoz, V, (1989) dir. Diagnóstico, Evaluación y Toma de decisiones.

Gómez, G., Flores, J, Giménez, E. (1996). Metodología de la Investigación Cualitativa. Barcelona: Tauro. 
Guerra López, Ingrid. (2007). Evaluación y Mejora Continua. Conceptos y herramientas para la medición y mejora del desempeño. Global Business Press. United States of America.

Hernández Sampieri, H y otros (2003). “Metodología de la Investigación”. Editorial: McGraw-Hill. México.

Hernández Sampieri, R. (2005 ).Metodología de la Investigación. La Habana: Félix Varela.

Hernández, Roberto; Fernández, Carlos y Baptista, Pilar. (1998), Metodología de la Investigación. $2^{\circ}$ Ed. Colombia. McGraw - Hill. 1998.

Lemus, Luis Arturo. (1993). Evaluación del Rendimiento Escolar. Capéelas, Buenos Aires

Lukas Mujika, José Francisco. Y Santiago Etxebarría, Karlos. (2004). Evaluación Educativa. Psicología y educación. Alianza Editorial. Madrid.

Ministerio de Educación y Cultura Y CEDOC. (2001). Proyecto Reforma Joven. Capacitación a distancia sobre Reforma de la Educación Media.Asunción, Última Hora, 2001. 180 p.

Muñoz Cuenca, Gustavo Adolfo. (2008). Un Nuevo Paradigma. La Quinta Generación de Evaluación. Memorias del Octavo Foro de Evaluación Educativa, 22, 23 y 24 de octubre de 2008. Mérida-Yucatán-México.

Nevo, José María; (997), La Evaluación en el Proceso de Aprendizaje Perspectivas, núm. 19, enero-junio, 2007, pp. 15-30 Universidad Católica Boliviana San Pablo Cochabamba, Bolivia

Proyecto ALDA EDUCA. (2010). Material de apoyo extraído para el taller de Preclase 2010, para los docentes de las escuelas beneficiarias 2007. Fuente consultada: Fascículo de evaluación

Serrano de Moreno, María Stella. (1990). El Proceso de Enseñanza Aprendizaje. Universidad de Los Andes Consejo de Estudios de Postgrado. Consejo Editorial. Mérida-Venezuela. 